\title{
THE CENTER OF A CONVEX SET
}

\author{
TECK-CHEONG LIM
}

Let $X$ be a Banach space and $K$ a weakly compact convex nonvoid subset with normal structure [1]. Brodskii and Mil'man [1] constructed, using transfinite induction, a "center" of $K$ which is fixed by every isometry mapping $K$ onto $K$. In this note, we construct a unique "center" for a weakly compact convex nonvoid subset (not necessarily having normal structure) which is fixed by every affine isometry mapping $K$ into $K$. A similar theorem for weak* compact convex sets is also possible under some additional assumptions.

Construction. Let $K$ be a nonempty weakly compact convex subset of a Banach space. We shall define $C_{\alpha}$ for all ordinals $\alpha$ by transfinite induction. Set $C_{0}=K$. Let $\beta$ be an ordinal and suppose that $C_{\alpha}$ has been defined for $\alpha<\beta$ in such a way that (i) each $C_{\alpha}$ is a nonempty closed convex subset of $K$ and (ii) $C_{\alpha}$, $\alpha<\beta$, is decreasing. If $\beta$ is a limit ordinal, we set $C_{\beta}=\cap_{\alpha<\beta} C_{\alpha}$. Otherwise, let $\gamma$ be the predecessor of $\beta$ and let

$$
S_{\beta}=\left\{z \in C_{\gamma}: z=\frac{1}{2}(x+y) \text { for some } x, y \in C_{\gamma} \text { with }\|x-y\|=\frac{1}{2} \operatorname{diam} C_{\gamma}\right\} .
$$

Then we set $C_{\beta}=\overline{\mathrm{Co}} S_{\beta}$. Since $C_{\gamma}$ is the closed convex hull of its strongly exposed points (see [2]), it is easy to see that if card $C_{\gamma}>1, C_{\beta}$ contains no strongly exposed points of $C_{\gamma}$ and hence is a proper subset of $C_{\gamma}$. If card $C_{\gamma}=1, C_{\beta}=C_{\gamma}$. It follows that for sufficiently large ordinals $\delta, C_{\delta}$ are identical and consist of exactly one point which we call the center of $K$.

If $X$ is a Banach space such that the dual of every separable subspace of $X$ is separable, and $K$ is a nonempty weak* compact convex subset of $X^{*}$, then every weak* closed convex nonempty subset of $K$ is the weak* closed convex hull of its weak* strongly exposed points (see [5]-[8]). With appropriate changes, the prior construction applies to this situation; in particular, replacing $C_{\beta}$ by $\overline{\mathrm{Co}^{*}}\left(S_{\beta}\right)$, where -* denotes the weak* closure. Thus $K$ has a unique center.

THEOREM 1. Let $K$ be a nonempty weakly compact convex subset of a Banach space. The center of $K$ is a fixed point of every affine isometry mapping $K$ into $K$.

Proof. Note that in the construction, each $C_{\alpha}$ is mapped into itself by every affine isometry of $K$ into $K$.

Received by the editors April 21, 1980.

1980 Mathematics Subject Classification. Primary 46B20; Secondary 47H10. 
TheOREM 2. Let $X$ be a Banach space such that the dual of every separable subspace of $X$ is separable. Let $K$ be a weak* compact convex nonempty subset of $X^{*}$. The center of $K$ is a fixed point of every weak* continuous affine isometry mapping $K$ into $K$.

Proof. If $T$ is an affine isometry, then $T\left(\operatorname{Co} S_{\beta}\right) \subseteq \operatorname{Co~} S_{\beta}$. By the weak* continuity, $T\left(C_{\beta}\right)=T\left(\overline{\mathrm{Co}}^{*} S_{\beta}\right) \subseteq C_{\beta}$.

Remarks. 1. It also follows from the Ryll-Nardzewski fixed point theorem (see [4]) that the family of affine isometries on $K$ has a common fixed point (which is not necessarily the center). Our approach follows that of Namioka-Asplund [4].

2. The assumption of weak* continuity in Theorem 2 cannot be removed since Example 1 in [3] shows that there are fixed point free affine isometries.

\section{REFERENCES}

1. M. S. Brodskii and D. P. Mil'man, On the center of a convex set, Dokl. Akad. Nauk SSSR 59 (1948), 837-840. MR 9, 448.

2. J. Diestel and J. J. Uhl, Vector measures, Math. Surveys, no. 15, Amer. Math. Soc., Providence, R.I., 1977 (particularly Chapter 7).

3. T. C. Lim, Asymptotic centers and nonexpansive mappings in some conjugate Banach spaces, Pacific J. Math. (to appear).

4. I. Namioka and E. Asplund, A geometric proof of Ryll-Nardzewski's fixed point theorem, Bull. Amer. Math. Soc. 73 (1967), 443-445. MR 35 \# 799.

5. I. Namioka and R. R. Phelps, Banach spaces which are Asplund spaces, Duke Math. J. 42 (1975), 735-750. MR 52 \# 11544.

6. C. Stegall, The Radon-Nikodym property in conjugate Banach spaces, Trans. Amer. Math. Soc. 206 (1975), 213-223. MR 51 \# 10581.

7. Math. 29 (1978), 408-412.

8. J. J. Uhl, A note on the Radon-Nikodym theorem for Banach spaces, Rev. Roumaine Math. Pures Appl. 17 (1972), 113-115.

Department of Mathematics, University of Chicago, Chicago, Illinois 60637

Current address: Department of Mathematics, George Mason University, Fairfax, Virginia 22030 